\title{
Metal-centered photoluminescence as a tool for detecting phase transitions in $\mathrm{Eu}^{\mathrm{III}}$ - and $\mathrm{Tb}^{\mathrm{III}}$-containing metallomesogens
}

Stéphane Suárez, Daniel Imbert, Frédéric Gumy, Claude Piguet, and Jean-Claude G. Bünzli

Laboratory of Lanthanide Supramolecular Chemistry, Swiss Federal Institute of Technology

Lausanne, BCH 1402, 1015 Lausanne, Switzerland, and Department of Inorganic, Analytical and

Applied Chemistry, University of Geneva, 30 quai E. Ansermet, 1211 Geneva 4, Switzerland

Electronic Supplementary material

(12 pages) 
Table S1. Energy of the absorption (Loge; solution in THF at 295K) and emission bands (solid state, $77 \mathrm{~K}$ ) of the ligands.

\begin{tabular}{l|c|c|c}
\hline Compound & $\begin{array}{c}E / \mathrm{cm}^{-1} \\
\text { (absorption) }\end{array}$ & $\begin{array}{c}E / \mathrm{cm}^{-1} \\
{ }^{1} \pi \pi^{*}\end{array}$ & $\begin{array}{c}E / \mathrm{cm}^{-1} \\
{ }^{3} \pi \pi^{*}\end{array}$ \\
\hline $\mathbf{L}^{1}$ & $45040(4.71)$ & 30670 & 20760 \\
& $37450(4.42)$ & $29970^{\mathrm{a}}$ & \\
& $33900(4.15)$ & & \\
$\mathbf{L}^{2}$ & $45500(4.64)$ & not observed & 20620 \\
& $37590(4.31)$ & & \\
& $34010(4.05)$ & & \\
\hline
\end{tabular}

${ }^{\mathrm{a}}$ Shoulder

Table S2. Integrated and corrected relative intensities of the $\mathrm{Eu}\left({ }^{5} \mathrm{D}_{0} \rightarrow{ }^{7} \mathrm{~F}_{J}\right)$ transitions in $\mathbf{E u L} \mathbf{L}^{\mathbf{1}}$.

\begin{tabular}{|c|c|c|c|c|c|c|c|c|}
\hline \multirow[t]{2}{*}{$T / \mathrm{K}$} & $\tilde{v}_{e x} / \mathrm{cm}^{-1}$ & $\int$ & $\int_{0 \rightarrow 1}$ & $\int_{0 \rightarrow 2}$ & $\int$ & $\int$ & $\int$ & $\int$ \\
\hline & & \multicolumn{3}{|l|}{$0 \rightarrow 0$} & $0 \rightarrow 3$ & $0 \rightarrow 4$ & $0 \rightarrow 5$ & $0 \rightarrow 6$ \\
\hline \multirow[t]{4}{*}{10} & 21468 & 0.01 & 1.0 & 4.7 & 0.09 & 1.1 & \multirow[t]{4}{*}{0.03} & \multirow[t]{4}{*}{0.13} \\
\hline & 17266 & - & 1.0 & 3.3 & 0.13 & 1.5 & & \\
\hline & 17244 & - & 1.0 & 5.8 & 0.03 & 0.6 & & \\
\hline & 17235 & - & 1.0 & 5.5 & 0.04 & 0.5 & & \\
\hline \multirow[t]{2}{*}{295} & 21468 & 0.02 & 1.0 & 8.1 & 0.06 & 0.9 & \multirow[t]{2}{*}{0.03} & \multirow[t]{2}{*}{0.12} \\
\hline & 17278 & - & 1.0 & 10.0 & 0.09 & 0.9 & & \\
\hline
\end{tabular}


Table S3. Energy of the $\operatorname{Eu}\left({ }^{5} \mathrm{D}_{0}\right)$ level in a solid state sample of $\mathbf{E u} \mathbf{L}^{\mathbf{1}}$ from excitation spectra at 10 and $295 \mathrm{~K}$, upon analysis of the $\mathrm{Eu}\left({ }^{5} \mathrm{D}_{0} \rightarrow{ }^{7} \mathrm{~F}_{1,2}\right)$ transitions ( $\mathrm{sh}=$ shoulder).

\begin{tabular}{|c|c|c|c|}
\hline$T /$ & $\tilde{v}_{\mathrm{an}} / \mathrm{cm}^{-1}$ & $\tilde{v}_{\max } / \mathrm{cm}^{-1}$ & $\tilde{v}_{\max } / \mathrm{cm}^{-1}$ \\
\hline K & & Site I & Site II \\
\hline \multirow[t]{6}{*}{10} & $16197\left({ }^{7} \mathrm{~F}_{2}\right)$ & 17282 sh, 17268 & 17249 (IIb), 17242 (IIa), $17236 \mathrm{sh}$ \\
\hline & $16818\left({ }^{7} \mathrm{~F}_{1}\right)$ & 17266 & 17249 (IIb), 17245, 17242 (IIa), 17237 \\
\hline & & & $\operatorname{sh}$ \\
\hline & $16915\left({ }^{7} \mathrm{~F}_{1}\right)$ & $17266 \mathrm{sh}$ & 17248 (IIb), 17245, 17242 (IIa),17 236 \\
\hline & & & sh \\
\hline & $17008\left({ }^{7} \mathrm{~F}_{1}\right)$ & 17266 & \\
\hline \multirow[t]{2}{*}{295} & $16185\left({ }^{7} \mathrm{~F}_{2}\right)$ & 17277 & \\
\hline & $16862\left({ }^{7} \mathrm{~F}_{1}\right)$ & 17278 & \\
\hline
\end{tabular}

Table S4. Lifetimes of the Eu( $\left({ }^{5} \mathrm{D}_{0}\right)$ excited level (ms) in $\mathbf{E u L}^{1}$ under various excitation conditions (the analyzing wavelength is set on the maximum of the ${ }^{5} \mathrm{D}_{0} \rightarrow{ }^{7} \mathrm{~F}_{1,2}$ transitions); $2 \sigma$ is given within parentheses.

\begin{tabular}{|c|c|c|c|c|}
\hline \multirow[t]{2}{*}{$T / \mathrm{K}$} & \multirow[t]{2}{*}{$\tilde{v}_{\mathrm{ex}} / \mathrm{cm}^{-1}$} & \multirow[t]{2}{*}{$\tilde{v}_{\text {an }} / \mathrm{cm}^{-1}$} & \multicolumn{2}{|l|}{$\tau / \mathrm{ms}$} \\
\hline & & & Site I & Site II \\
\hline \multirow[t]{8}{*}{10} & 28170 (ligand) & $17008\left({ }^{7} \mathrm{~F}_{1}\right)$ & $1.05(4)$ & \\
\hline & & $16915\left({ }^{7} \mathrm{~F}_{1}\right)$ & & $0.51(4)$ \\
\hline & & $16818\left({ }^{7} F_{1}\right)$ & $1.02(6)$ & $0.43(2)$ \\
\hline & 21468 & $17008\left({ }^{7} F_{1}\right)$ & $1.09(2)$ & \\
\hline & & $16915\left({ }^{7} F_{1}\right)$ & & $0.58(4)$ \\
\hline & & $16818\left({ }^{7} F_{1}\right)$ & $1.12(4)$ & $0.43(2)$ \\
\hline & 17266 (site I) & $17008\left({ }^{7} F_{1}\right)$ & $1.05(2)$ & \\
\hline & 17242 (site II) & $16915\left({ }^{7} F_{1}\right)$ & & $0.51(4)$ \\
\hline \multirow[t]{7}{*}{295} & 28170 (ligand) & $16851\left({ }^{7} F_{1}\right)$ & $0.73(6)$ & \\
\hline & & $16187\left({ }^{7} F_{2}\right)$ & $0.72(4)$ & \\
\hline & 21468 & $16851\left({ }^{7} F_{1}\right)$ & $0.66(5)$ & \\
\hline & & $16187\left({ }^{7} F_{2}\right)$ & $0.75(8)$ & \\
\hline & 17278 & $16185\left({ }^{7} F_{1}\right)$ & $0.82(2)$ & \\
\hline & & $14591\left({ }^{7} \mathrm{~F}_{4}\right)$ & $0.77(8)$ & \\
\hline & & $14591\left({ }^{7} F_{4}\right)$ & $0.77(9)$ & \\
\hline
\end{tabular}


Table S5. Lifetimes of the $\mathbf{T b}\left({ }^{5} \mathrm{D}_{4}\right)$ excited level (ms) in $\mathbf{T} \mathbf{b} \mathbf{L}^{\mathbf{1}}$ under various excitation conditions (analyzing wavelength set on the maximum of the ${ }^{5} \mathrm{D}_{4} \rightarrow{ }^{7} \mathrm{~F}_{6,5,4}$ transitions); $2 \sigma$ is given within parentheses.

\begin{tabular}{cccc}
\hline$T / \mathrm{K}$ & $\tilde{v}_{\mathrm{ex}} / \mathrm{cm}^{-1}$ & $\tilde{v}_{\mathrm{an}} / \mathrm{cm}^{-1}$ & $\tau(\mathrm{ms})$ \\
\hline 10 & 28170 (Ligand) & $20411\left({ }^{7} \mathrm{~F}_{6}\right)$ & $1.18(2)$ \\
& & $18372\left({ }^{7} \mathrm{~F}_{5}\right)$ & $1.13(2)$ \\
& 20492 & $18372\left({ }^{7} \mathrm{~F}_{5}\right)$ & $1.06(4)$ \\
& & $17063\left({ }^{7} \mathrm{~F}_{4}\right)$ & $1.09(4)$ \\
& \multirow{2}{*}{28170 (Ligand) } & $20403\left({ }^{7} \mathrm{~F}_{6}\right)$ & $1.09(2)$ \\
& & $18382\left({ }^{7} \mathrm{~F}_{5}\right)$ & $1.08(2)$ \\
& 20403 & $18382\left({ }^{7} \mathrm{~F}_{5}\right)$ & $1.19(2)$ \\
& & $17062\left({ }^{7} \mathrm{~F}_{4}\right)$ & $1.09(2)$
\end{tabular}

Table S6. Integrated and corrected relative intensities of the $\operatorname{Eu}\left({ }^{5} \mathrm{D}_{0} \rightarrow{ }^{7} \mathrm{~F}_{J}\right)$ transitions in $\mathbf{E u} \mathbf{L}^{\mathbf{1}}$ at various temperatures.

\begin{tabular}{ccccc}
\hline & ${ }^{7} \mathrm{~F}_{0} /$ & ${ }^{7} \mathrm{~F}_{1} /$ & ${ }^{7} \mathrm{~F}_{2} /$ & ${ }^{7} \mathrm{~F}_{4} /$ \\
\hline 298 & $\Sigma\left({ }^{7} \mathrm{~F}_{j}\right)$ & $\Sigma\left({ }^{7} \mathrm{~F}_{j}\right)$ & $\Sigma\left({ }^{7} \mathrm{~F}_{j}\right)$ & $\Sigma\left({ }^{7} \mathrm{~F}_{j}\right)$ \\
323 & 0.002 & 0.07 & 0.88 & 0.05 \\
343 & 0.001 & 0.07 & 0.88 & 0.05 \\
348 & 0.001 & 0.07 & 0.89 & 0.05 \\
353 & 0.001 & 0.06 & 0.89 & 0.05 \\
358 & 0.001 & 0.06 & 0.89 & 0.05 \\
363 & 0.001 & 0.06 & 0.89 & 0.05 \\
373 & 0.001 & 0.05 & 0.90 & 0.05 \\
393 & 0.001 & 0.06 & 0.89 & 0.05 \\
383 & 0.003 & 0.06 & 0.89 & 0.05 \\
373 & 0.001 & 0.06 & 0.89 & 0.05 \\
363 & 0.002 & 0.06 & 0.88 & 0.05 \\
353 & 0.002 & 0.06 & 0.89 & 0.05 \\
343 & 0.001 & 0.06 & 0.89 & 0.05 \\
323 & 0.002 & 0.06 & 0.88 & 0.05 \\
& & & &
\end{tabular}




$$
\begin{array}{lllll}
298 & 0.002 & 0.06 & 0.88 & 0.06
\end{array}
$$

Table S7. Lifetimes of the $\mathrm{Eu}\left({ }^{5} \mathrm{D}_{0}\right)$ excited level $(\mathrm{ms})$ in $\mathbf{E u L}^{1}$ under various excitation conditions (broad band at $355 \mathrm{~nm}$ and selective excitation at $579.7 \mathrm{~nm}$; the analyzing wavelength is set on the maximum of the ${ }^{5} \mathrm{D}_{0} \rightarrow{ }^{7} \mathrm{~F}_{2}$ transition); $2 \sigma$ is $0.01-0.02$.

\begin{tabular}{|c|c|c|c|c|c|c|c|}
\hline & $T / \mathrm{K}$ & $\begin{array}{c}\lambda_{\mathrm{ex}}= \\
355 \mathrm{~nm}\end{array}$ & $\begin{array}{c}\lambda_{\mathrm{ex}}= \\
579.7 \\
\mathrm{~nm}\end{array}$ & & $T / \mathrm{K}$ & $\begin{array}{c}\lambda_{\mathrm{ex}}= \\
355 \mathrm{~nm}\end{array}$ & $\begin{array}{c}\lambda_{\mathrm{ex}}= \\
579.7 \\
\mathrm{~nm}\end{array}$ \\
\hline \multirow{15}{*}{$\uparrow$} & 298 & 0.62 & 0.62 & $\uparrow$ & 403 & 0.26 & 0.27 \\
\hline & 323 & 0.59 & 0.61 & $\downarrow$ & 323 & 0.58 & 0.59 \\
\hline & 333 & 0.56 & 0.59 & $\uparrow$ & 403 & 0.27 & 0.27 \\
\hline & 343 & 0.51 & 0.57 & $\downarrow$ & 323 & 0.59 & 0.58 \\
\hline & 346 & 0.49 & 0.55 & $\uparrow$ & 403 & 0.25 & 0.26 \\
\hline & 349 & 0.47 & 0.52 & $\downarrow$ & 323 & 0.58 & 0.59 \\
\hline & 352 & 0.44 & 0.5 & $\uparrow$ & 403 & 0.28 & 0.26 \\
\hline & 355 & 0.41 & 0.48 & $\downarrow$ & 323 & 0.57 & 0.57 \\
\hline & 358 & 0.39 & 0.44 & $\uparrow$ & 403 & 0.26 & 0.27 \\
\hline & 361 & 0.36 & 0.41 & $\downarrow$ & 323 & 0.58 & 0.59 \\
\hline & 364 & 0.33 & 0.35 & $\uparrow$ & 403 & 0.28 & 0.27 \\
\hline & 367 & 0.31 & 0.32 & & & & \\
\hline & 373 & 0.29 & 0.3 & & & & \\
\hline & 383 & 0.28 & 0.29 & & & & \\
\hline & 403 & 0.27 & 0.28 & & & & \\
\hline \multirow{7}{*}{$\downarrow$} & 383 & 0.29 & 0.3 & & & & \\
\hline & 373 & 0.3 & 0.32 & & & & \\
\hline & 363 & 0.34 & 0.36 & & & & \\
\hline & 353 & 0.41 & 0.42 & & & & \\
\hline & 343 & 0.48 & 0.5 & & & & \\
\hline & 323 & 0.55 & 0.57 & & & & \\
\hline & 298 & 0.61 & 0.63 & & & & \\
\hline
\end{tabular}




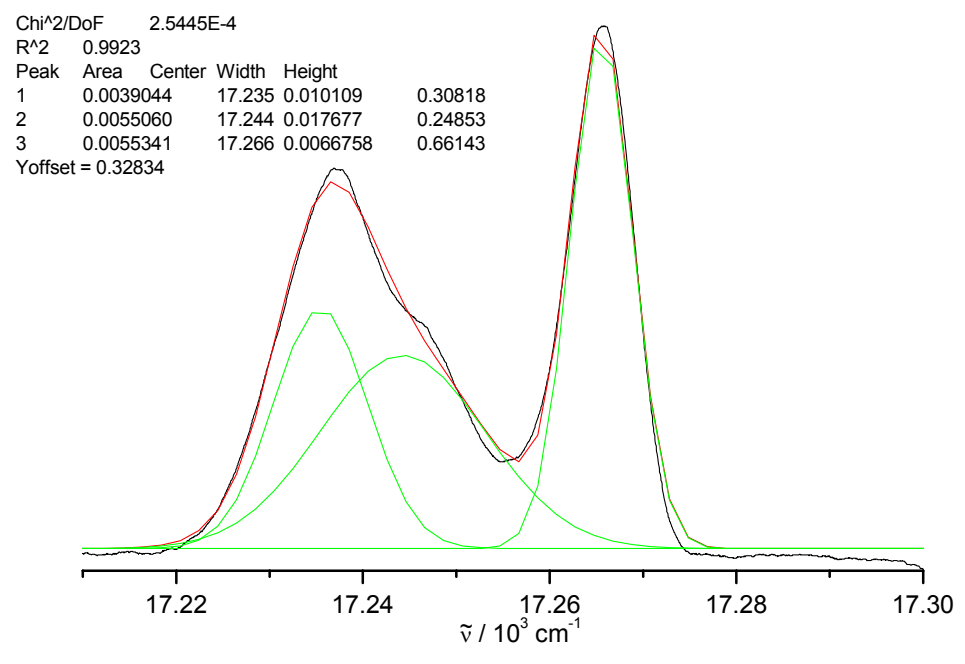

Figure S1. Decomposition of the spectrum of the ${ }^{5} \mathrm{D}_{0} \rightarrow{ }^{7} \mathrm{~F}_{0}$ transition at $10 \mathrm{~K}\left(\tilde{v}_{\mathrm{ex}}=24468 \mathrm{~cm}^{-1}\right)$.

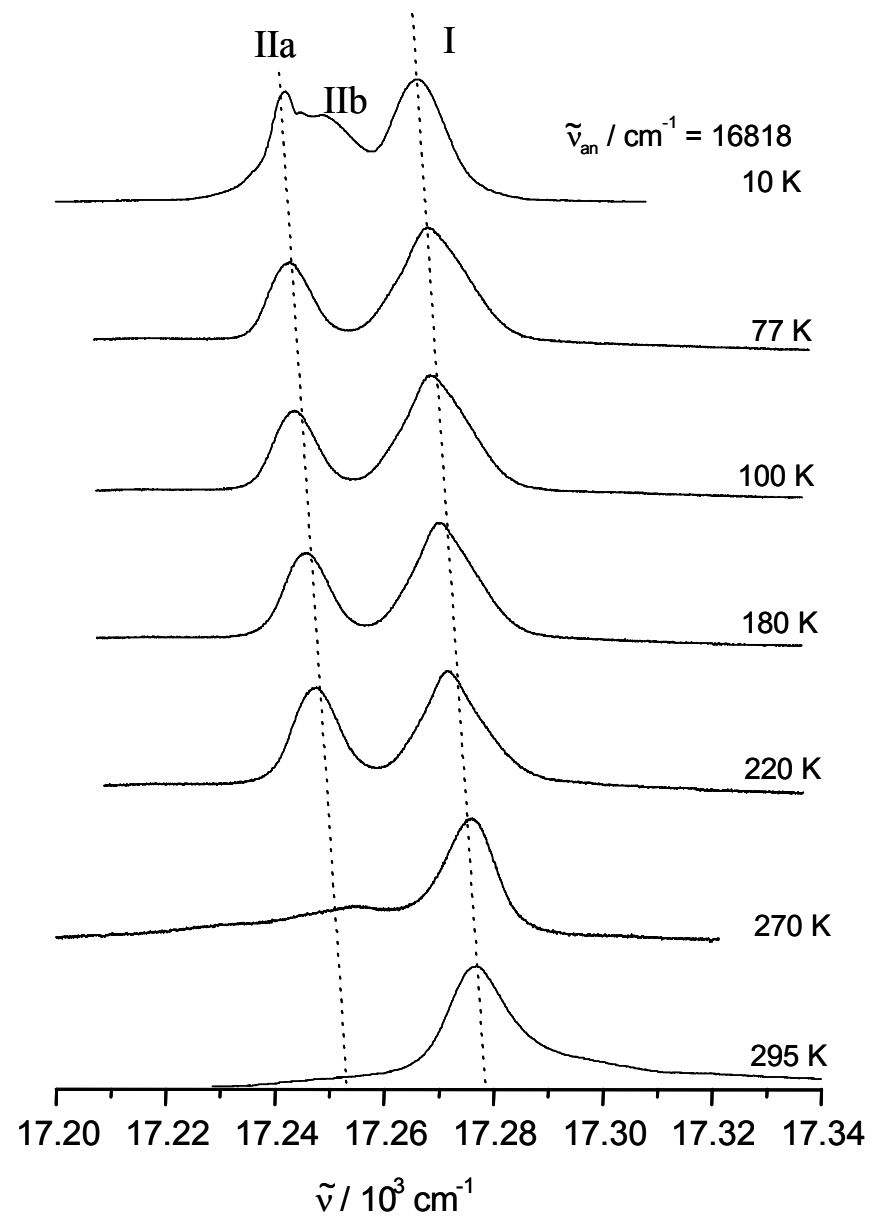

Figure S2. Excitation spectra of a solid state sample of $\mathbf{E} \mathbf{u} \mathbf{L}^{1}$ upon monitoring the $\operatorname{Eu}\left({ }^{5} \mathrm{D}_{0} \rightarrow{ }^{7} \mathrm{~F}_{1}\right)$ transition, as a function of temperature. 
a)

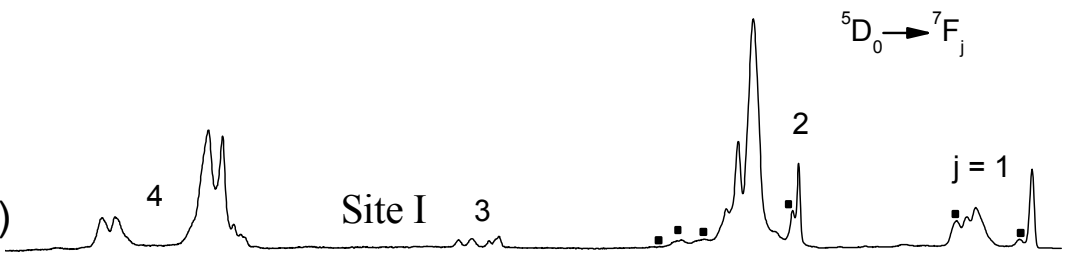

b)

Site IIb
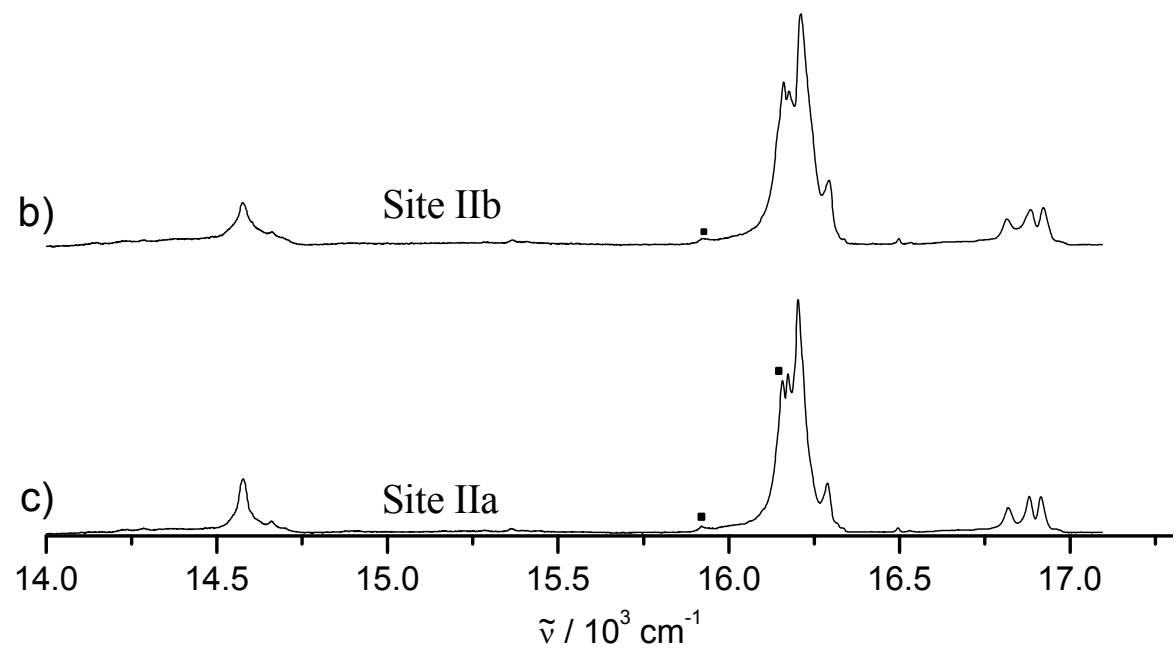

Figure S3. High-resolution emission spectra of a solid state sample of $\mathbf{E} \mathbf{u L}^{\mathbf{1}}$ recorded at $10 \mathrm{~K}$ upon selective excitation (a) $\tilde{v}_{\mathrm{ex}}=17266 \mathrm{~cm}^{-1}$, (b) $17250 \mathrm{~cm}^{-1}$, (c) $17242 \mathrm{~cm}^{-1}$; $\mathbf{\square}$ : vibronic transition.
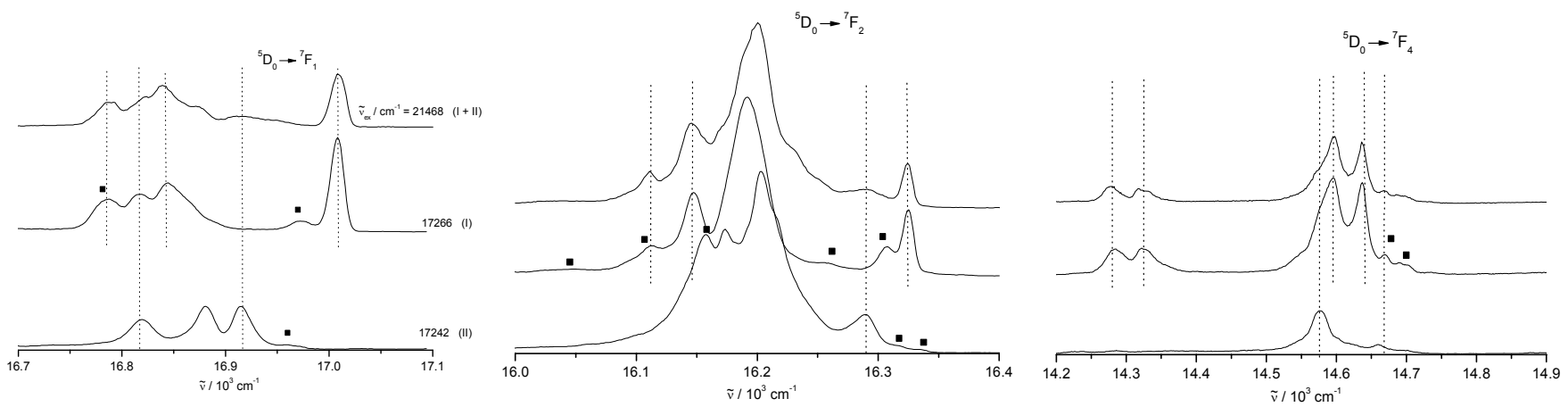

Figure S4. Emission spectra of $\mathbf{E u} \mathbf{L}^{1}$ in the solid state at $10 \mathrm{~K}$, upon broad band and selective excitation; $\mathbf{\square}$ : vibronic transition. 

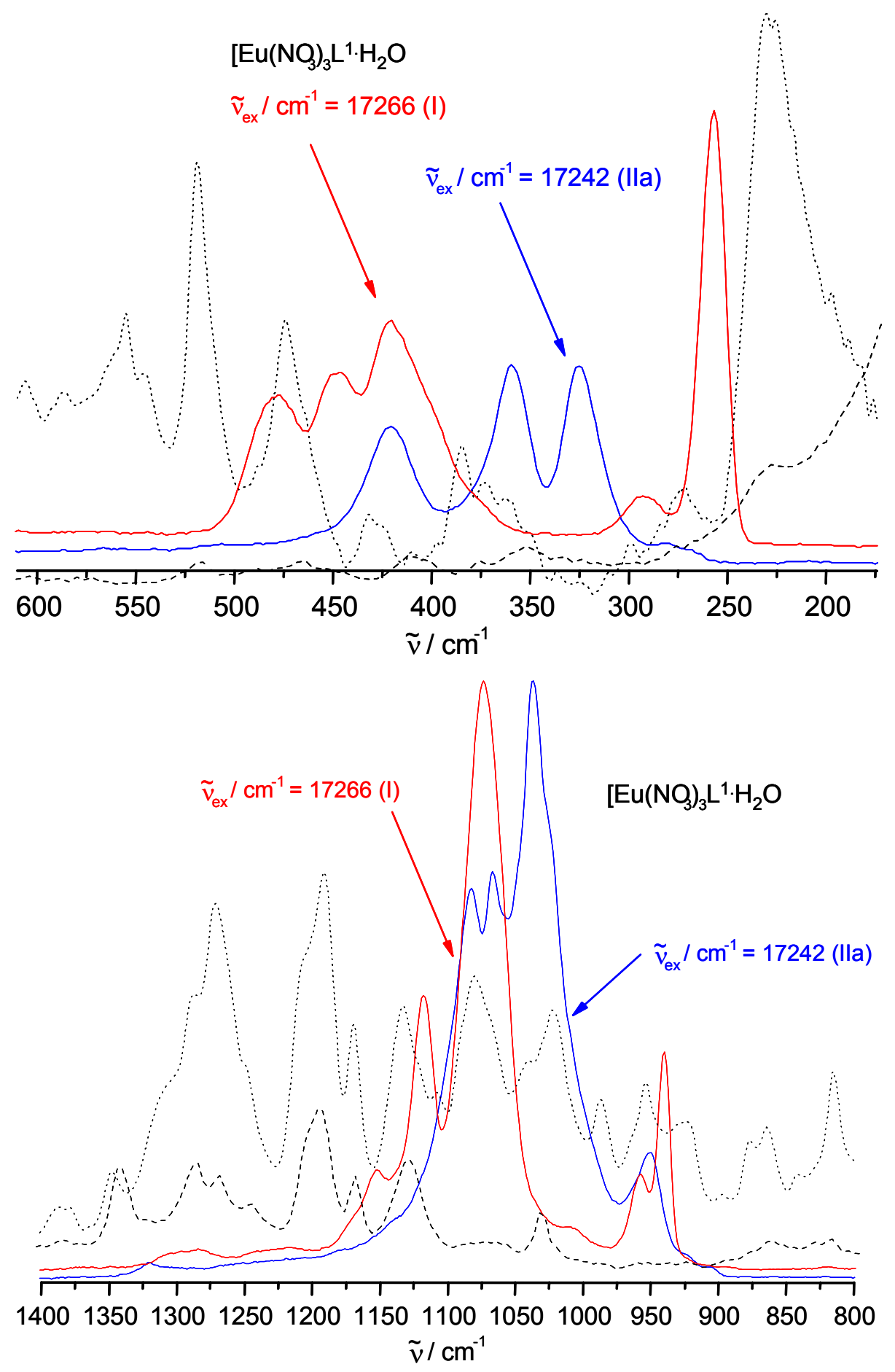

Figure S5. Comparison between emission spectra $\left(\tilde{v}_{e x}=17266,17242 \mathrm{~cm}^{-1}\right.$, solid line), IR (dotted line) and Raman (dashed line) spectra of $\mathbf{E} \mathbf{u} \mathbf{L}^{1}$ in the energy range of the ${ }^{5} \mathrm{D}_{0} \rightarrow{ }^{7} \mathrm{~F}_{1}$ (top) and ${ }^{5} \mathrm{D}_{0} \rightarrow{ }^{7} \mathrm{~F}_{2}$ (bottom) transitions 


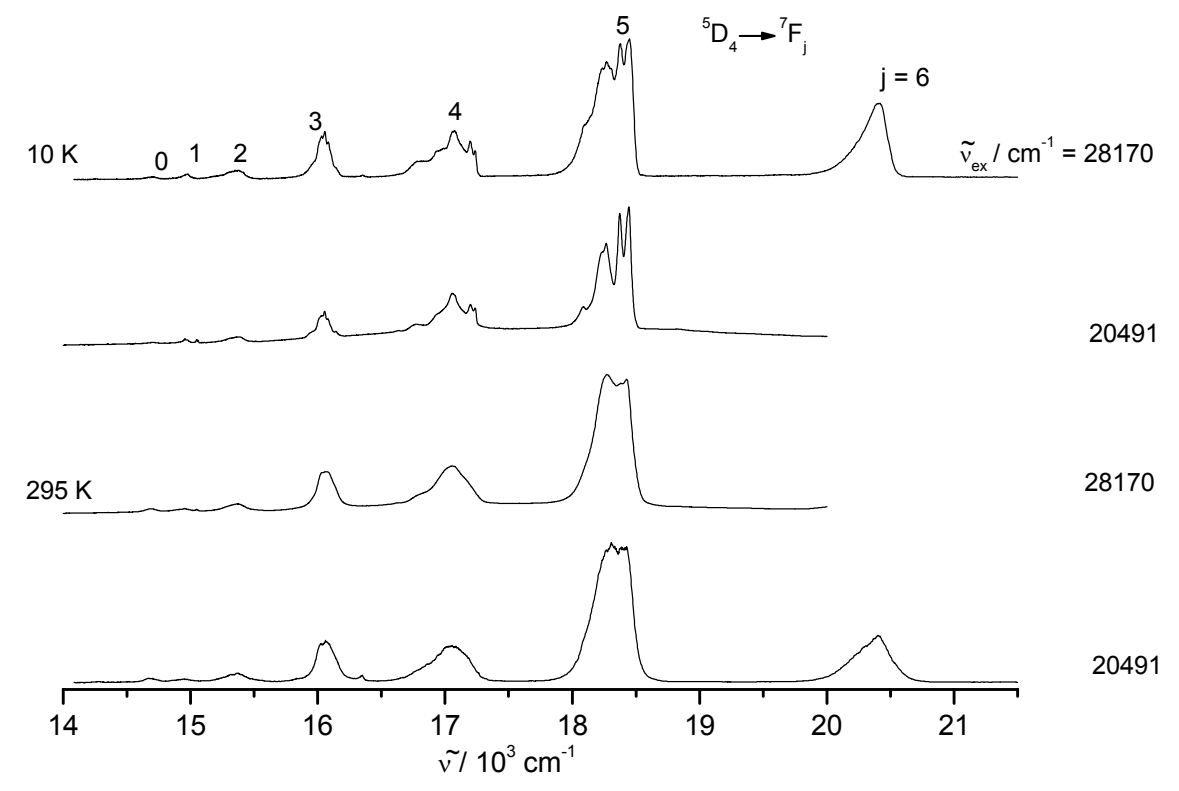

Figure S6. Emission spectra of the $\mathbf{T b} \mathbf{L}^{\mathbf{1}}$ complex at 10 and $295 \mathrm{~K}$ in the solid state under various excitation conditions.

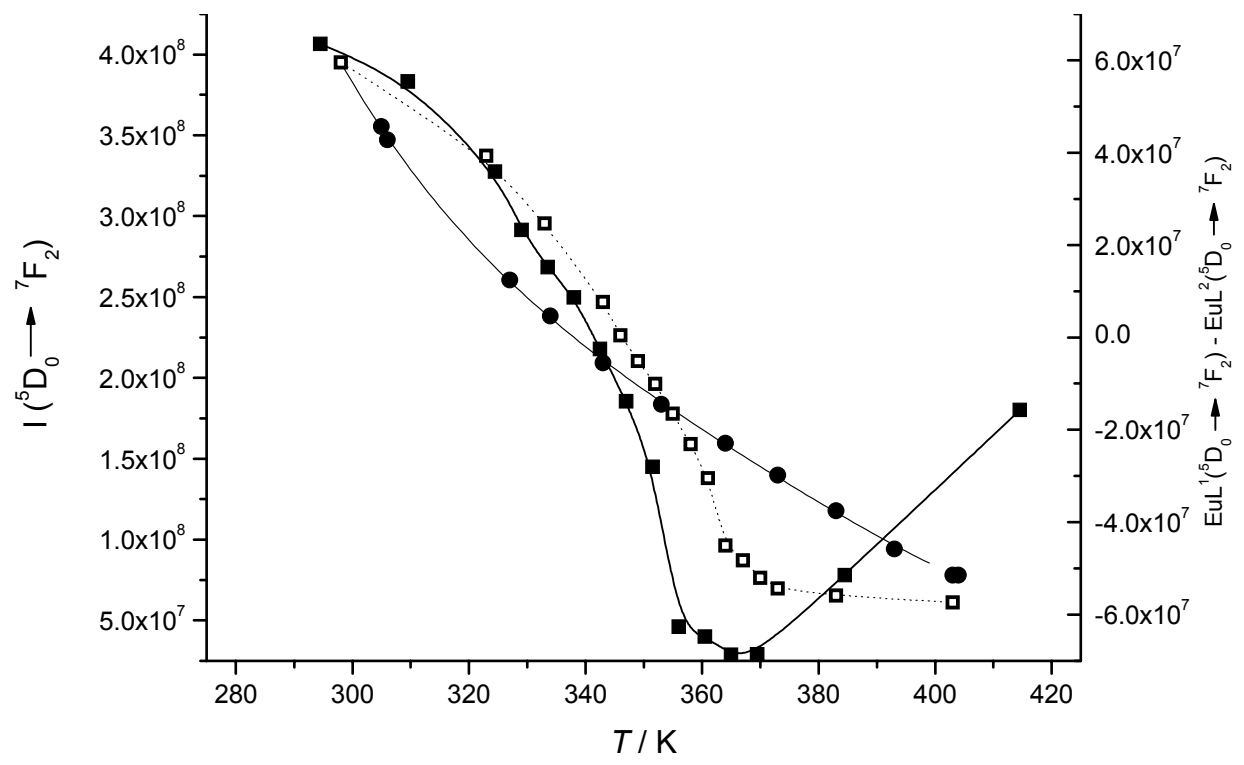

Figure S7: (Left scale, from 403 to $298 \mathrm{~K}$, excitation at $28170 \mathrm{~cm}^{-1}$ ): Calculated curve of the EuL ${ }^{1}$ intensity from experimental values with the maximum of $\mathbf{E} \mathbf{u} \mathbf{L}^{2}(\square)$; Calculated curve with a $4^{\text {th }}$-order polynomial ( - ) and experimental points (O) of the EuL ${ }^{1}$ intensity. (Right scale, $\mathbf{\square}$ ): $\mathbf{E u L} \mathbf{L}^{\mathbf{1}}-\mathbf{E} \mathbf{\mathbf { u }} \mathbf{L}^{\mathbf{2}}$. 


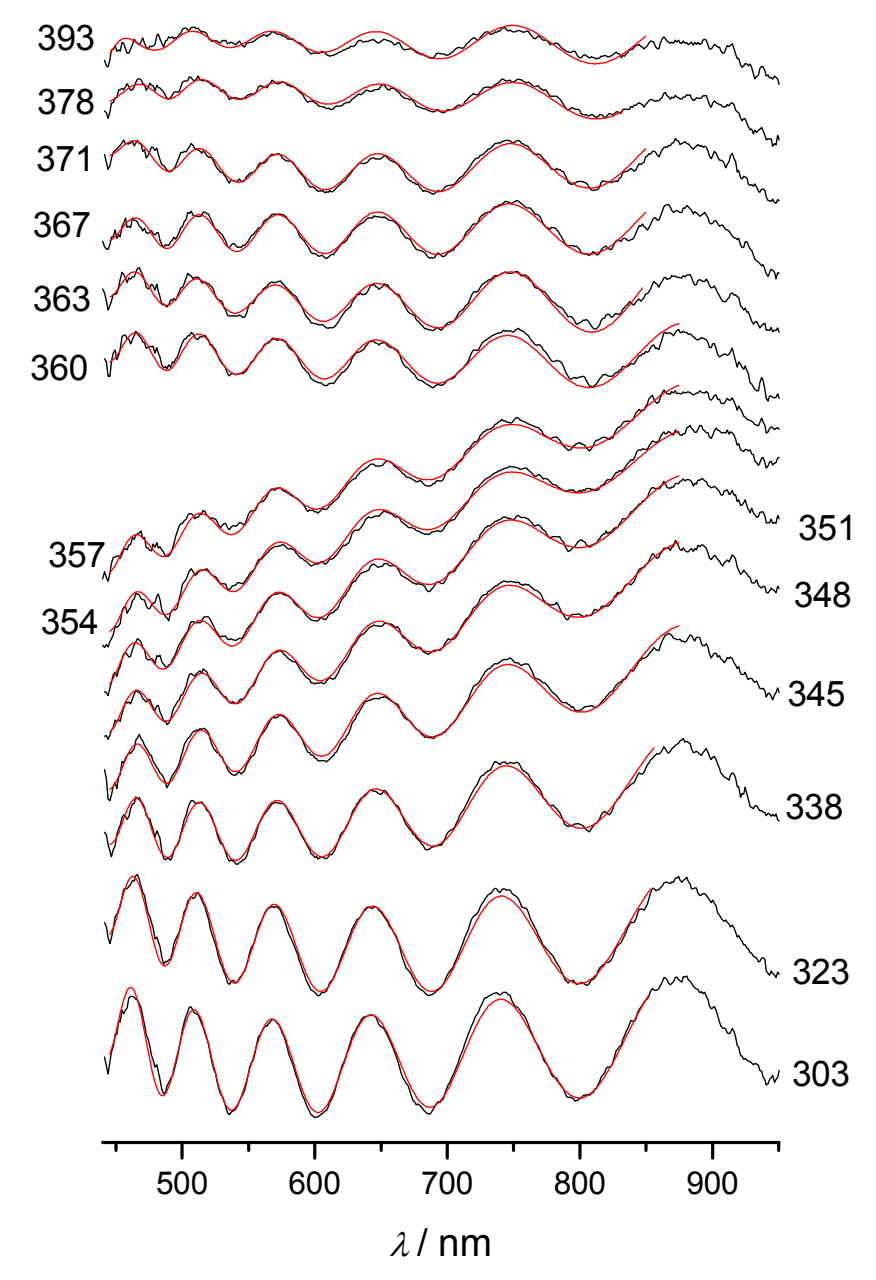

Figure S8. Measured ( - ) and calculated ( - ) reflectance spectra of the films of $\mathbf{E u L}^{\mathbf{1}}$ from 303 to $393 \mathrm{~K}$. 


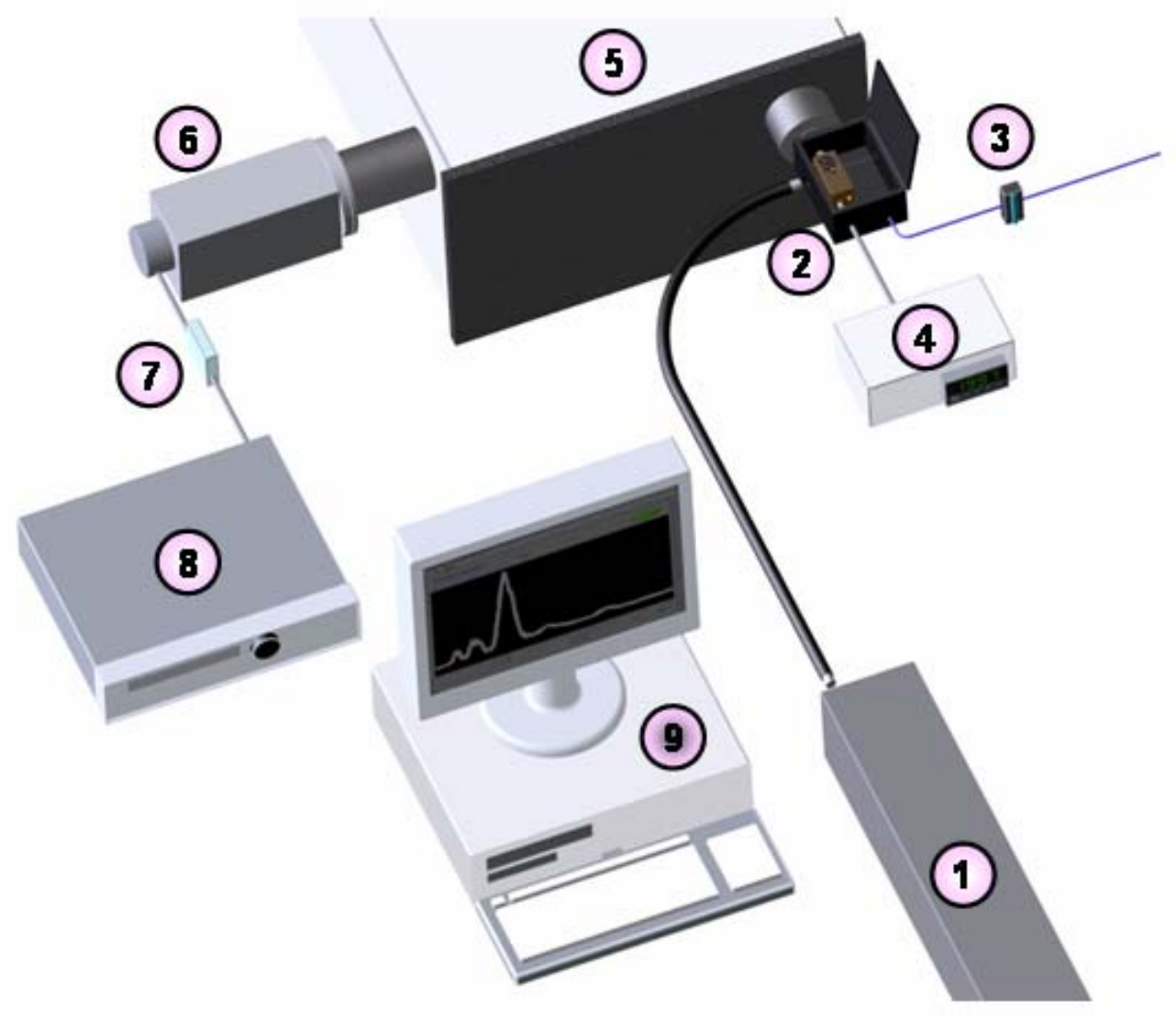

Figure S9. Schematic description of the equipment used for monitoring the phase transition by recording luminescence parameters: (1) Excitation laser, (2) Thermostated sample holder, (3) Argon flux controller, (4) Command relay, (5) Double monochromator (Spex 1404), (6) Photomultiplier (S20 cathode), (7) Linear amplifier (500 MHz), (8) Photon counter (emission spectra)/Multichannel scaler (without (7), lifetime measurements), (9) Data treatment (PC). 


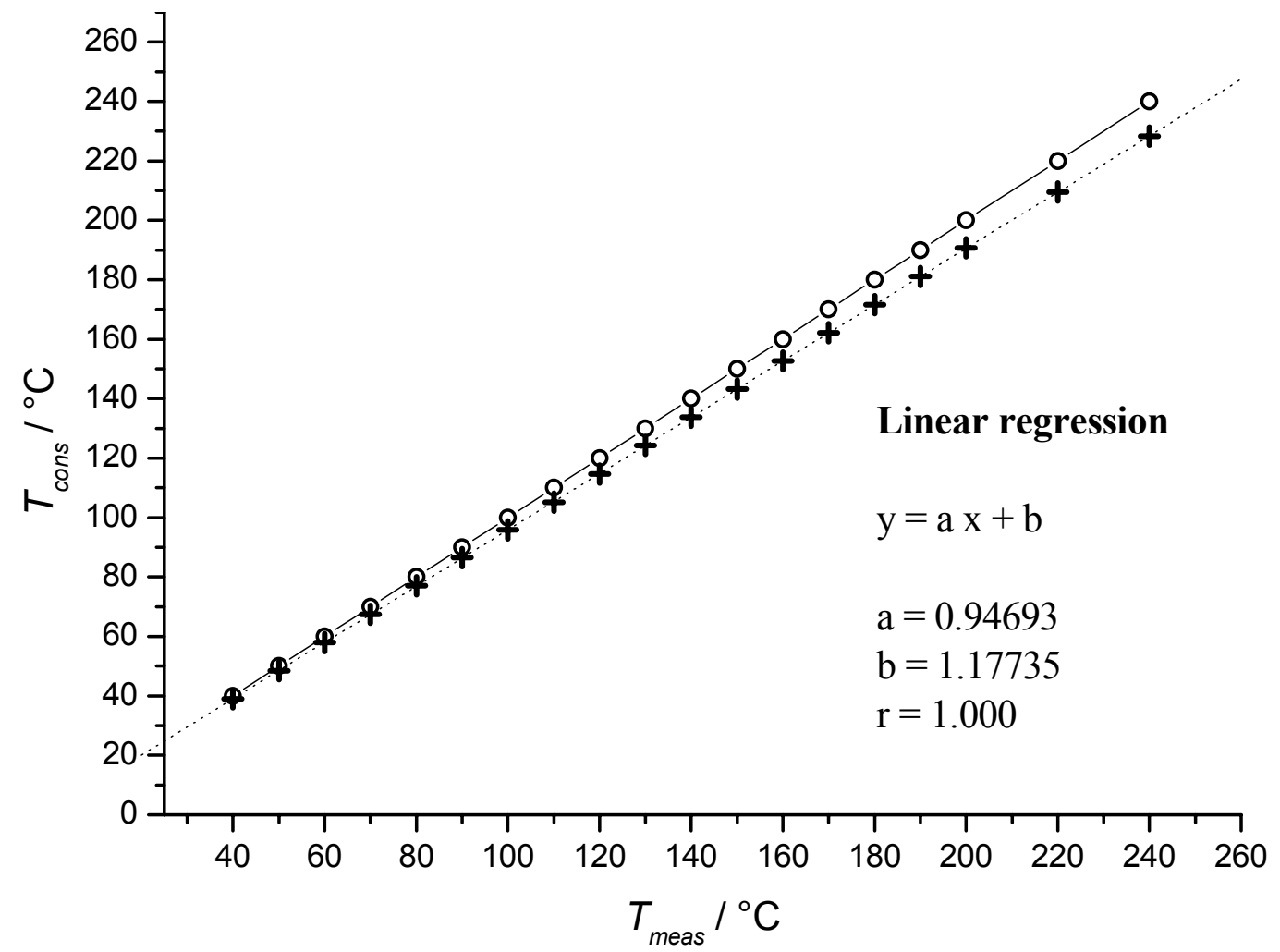

Figure S10. Preset $T_{\text {cons }}(\mathrm{O})$ and measured $T_{\text {meas }},(+)$ temperatures of the sample (see text for explanation). 